\title{
Challenges and Opportunities in Marginal Zone Lymphoma: Implications of Biology on Treatment
}

\author{
Presented by Stephen D. Smith, MD
}

\begin{abstract}
Although sharing some common features in terms of etiology and even clinical presentation, marginal zone lymphoma is a heterogeneous group of diseases, with subtypes that can present in unexpected ways, especially with regard to autoimmune disease. There's a proven role of antimicrobial therapy for both Helicobacter pylori and hepatitis $\mathrm{C}$ virus, and 10-year overall survival is high. Because this disease requires continuous treatment, therapy should be properly sequenced, with consideration given to patient comorbidities and age. Finally, B-cell receptor signaling inhibitors and immunomodulatory agents can provide responses and disease control.
\end{abstract}

Marginal zone lymphoma (MZL) is a heterogeneous group of diseases, but they share common features in terms of etiology and even clinical presentation. Given favorable rates of long-term survival, sequencing and therapy selection play an important role over the "marathon" course of the disease, according to Stephen D. Smith, MD, Associate Professor, University of Washington/Fred Hutchinson Cancer Research Center. At the NCCN 2020 Virtual Congress: Hematologic Malignancies, Dr. Smith discussed the etiology of MZL, current treatment standards by subtype, and the role of targeted therapies (Figure 1).

\section{General Principles of First-Line Treatment}

Similar to follicular lymphoma, indications for treatment of MZL follow the modified GELF (Groupe d'Etude des Lymphomes Folliculaires) criteria and require a "good degree of clinical judgment," said Dr. Smith, who suggested consulting a rheumatologist in some cases.

According to Dr. Smith, it is essential to treat infection in patients with Helicobacter pylori-positive gastric MZL and those with hepatitis $\mathrm{C}$ virus and any MZL. In addition, clinicians may consider treating infection in orbital mucosa-associated lymphoid tissue (MALT) lymphoma.

Standard first-line therapies include involved-site radiotherapy (ISRT) for localized disease. Localized fields are better than wide fields, especially for parotid MALTs, said Dr. Smith, noting that cautiously working with a radiation oncologist to apply ISRT can yield longterm remissions. Finally, chemoimmunotherapy is reserved for patients with advanced-stage disease or disseminated nodal involvement.

\section{Gastric MALT Lymphoma}

For patients with gastric MALT lymphoma and positive $H$ pylori infection at baseline, the infection should be eradicated with a triple therapy of proton pump inhibitor + clarithromycin + amoxicillin. If the tumor is positive for translocation $(11 ; 18)$ or the infection fails to respond to initial therapy, clinicians should consider adding ISRT or rituximab if RT is not an option.

With distant nodes or systemic involvement, treatment for gastric MALT lymphoma is systemic chemoimmunotherapy with an alkylator + rituximab or single-agent rituximab for the elderly or infirm. A trial of anthracycline demonstrated no real benefits in this patient population., "Certainly, anthracyclinecontaining therapy is an option for subsets of patients, but in my general practice, it's chemoimmunotherapy with rituximab and an alkylating agent for first-line treatment in the vast majority of patients," Dr. Smith explained.

\section{Nongastric MALT Lymphoma}

With respect to nongastric MALT sites (eg, thyroid, breast, orbits), ISRT can be considered; however, it is also safe to observe patients if surgical treatment is successful, said Dr. Smith.

For advanced or relapsed/refractory disease, chemoimmunotherapy has been demonstrated to improve outcomes more than chemotherapy alone. This therapy 


\begin{tabular}{|c|c|c|c|}
\hline & MALT Lymphoma & Nodal MZL & Splenic MZL \\
\hline \multirow[t]{2}{*}{ Diagnostic evaluation } & \multicolumn{3}{|c|}{$\begin{array}{l}\text { - Imaging (CT, consider whole-body PET but not always FDG-avid) } \\
\text { - Biopsy of affected site, labs (CBC diff, LDH, SPEP, HCB/HCV, HIV) }\end{array}$} \\
\hline & $\begin{array}{l}\text { Endoscopic evaluation } \\
\text { H pylori (histopathology, } \\
\text { breath, stool- gastric) }\end{array}$ & Akin to FL (a nodal disease) & $\begin{array}{c}\text { Spleen/marrow predominant } \\
\text { BM aspirate and biopsy may } \\
\text { suffice }\end{array}$ \\
\hline \multirow[t]{2}{*}{ Pathologic findings } & \multicolumn{3}{|c|}{$\begin{array}{c}\text { Clonal population, positive for B-cell markers CD19, CD20, and CD22; } \\
\text { negative for } C D 5, C D 10, C D 23\end{array}$} \\
\hline & $\begin{array}{c}\text { Resemble MALT tissue: } \\
\text { variable infiltrate: small } \\
\text { lymphocytes, monocytoid } \\
\text { B-cells, marginal zone } \\
\text { (centrocyte-like) B-cells, and } \\
\text { plasma cells }\end{array}$ & $\begin{array}{l}\text { - Surface markers differ } \\
\text { from FL, MCL, CLL } \\
\text { - Unique "monocytoid B-cell } \\
\text { morphology" } \\
\text { - } \text { MYD88 mutation usually }_{\text {neg }^{\mathrm{a}}}\end{array}$ & $\begin{array}{l}\text { - Surface markers (Surface } \\
\text { lg) } \\
\text { - Morphology may include } \\
\text { "villous" projections } \\
\text { - Nodular marrow } \\
\text { involvement }\end{array}$ \\
\hline
\end{tabular}

Figure 1. Diagnosis of MZLs.

Abbreviations: BM, bone marrow; CLL, chronic lymphocytic leukemia; HBV, hepatitis B virus; HCV, hepatitis C virus; FL, follicular lymphoma; MALT, mucosa-associated lymphoid tissue; $M C L$, mantle cell lymphoma; $M Z L$, marginal zone lymphoma.

aMYD88 L265P mutation can be present in MZL (10\%-20\%).

can also be delivered in a shortened format. Results of a randomized trial of patients with MALT lymphoma showed improved event-free survival and progression-free survival (PFS) with rituximab + chlorambucil versus either chlorambucil or rituximab monotherapy. ${ }^{3}$ In a separate study of patients with MALT lymphoma, first-line response-adapted treatment with combination bendamustine/rituximab yielded an $88 \%$ event-free survival at 4 years. ${ }^{4}$ "This is a nice, disease-specific dataset that provides justification for 4 cycles of bendamustine/rituximab in MALT, assuming that patients achieve a complete response after the first 3," said Dr. Smith.

\section{Nodal MZL}

For nodal MZLs, the pattern of treatment is very similar: ISRT for limited-stage disease, chemoimmunotherapy for advanced-stage disease, and rituximab for elderly or infirm patients.

Results of the E4402 RESORT study showed that maintenance rituximab significantly improved time to treatment failure in small lymphocytic lymphoma and MZL compared with a retreatment dosing strategy. ${ }^{5}$ Although there was no overall survival benefit, said Dr. Smith, this study demonstrated that the need for cytotoxic chemotherapy can be delayed. "Considering extended dosing of rituximab, especially for responders who receive single-agent rituximab, is a very reasonable approach," he observed.

\section{Splenic MZL}

Compared with the other subtypes, splenic MZL is most frequently associated with hepatitis $C$ virus, which should be evaluated and treated if present. This subtype is also associated with high virologic and tumor response rates. ${ }^{6}$
Single-agent rituximab is also "strikingly effective" in this subtype, said Dr. Smith, and there have been data suggesting superiority to splenectomy alone. ${ }^{7}$ "I would reserve splenectomy for treatment on relapse, if needed, or if there's great diagnostic uncertainty or suspicion for transformation," he said.

\section{Pathobiology and Potential Treatment Targets}

As Dr. Smith explained, the pathogenesis of MZL arises from the cooperation of immunologically driven and genetic changes in a multistep process, the timing of which is not always clear. In some forms of the disease, however, antigenic stimulation drives formation of the clone, followed by genetic and epigenetic lesions that accumulate and determine malignant behavior.

The pathobiology of the disease is also indicative of potential treatment targets. Chronic antigenic stimulation suggests the potential to modulate the microenvironment, and lenalidomide has been shown to do that and has demonstrated efficacy. B-cell receptor activation, which is related to NF-kappa B activation, can be inhibited with inhibitors of BTK and/or PI3K. Finally, there are epigenetic lesions, which could be emerging and feasible targets in the future.

\section{Ibrutinib}

Ibrutinib was FDA-approved in 2018 for relapsed/refractory MZL based on pivotal data that showed an overall response rate of $48 \%$ when it was given as a continuous therapy. ${ }^{8}$ Median PFS was 14 months on ibrutinib, but differed by subtypes. Splenic MZL performed the best of all subtypes, with a median PFS of 19.4 months.

The safety of ibrutinib in MZL is similar to that seen in other diseases. Frequently occurring adverse effects 
include fatigue, diarrhea, and anemia. Atrial fibrillation should be a reason for concern in patients with cardiac history, said Dr. Smith. Otherwise, there was no signal for excess toxicity.

\section{Lenalidomide}

Lenalidomide is typically given in combination with rituximab at a dose of $20 \mathrm{mg}$ for 3 out of 4 weeks. Treatment duration is varied, with either the patient achieving complete response or treatment continuing for 8 to 12 months. As Dr. Smith explained, it is harder to keep patients on lenalidomide for many years because of its toxicity, but when combined with rituximab, most patients will respond. Response rates range from $54 \%$ to $80 \% .^{9-11}$ Although based on small, single-arm studies, PFS estimates range from 17 months to $>2$ years. ${ }^{9-11}$ "Patients can achieve durable benefit with a course of lenalidomide and rituximab in MZL," said Dr. Smith, who typically uses it in the relapsed/refractory setting. "It's certainly a reasonable treatment option."

The AUGMENT trial showed no difference in PFS between placebo/rituximab and lenalidomide/rituximab, but this subset was underpowered. ${ }^{11}$ "This was not a perfect study, but it does suggest that when clinicians utilize lenalidomide in MZL, we should do so with a grain of salt and make sure that the patient has had a good try at single-agent rituximab first or in continued therapy before trying lenalidomide," said Dr. Smith.

Nevertheless, modern treatment guidelines from $\mathrm{NCCN}^{12}$ and $\mathrm{ESMO}^{13}$ include lenalidomide + rituximab as a treatment option for MALT lymphoma and other subtypes.

\section{PI3K Inhibitors}

When used to treat patients with MZL, PI3K inhibitors are selected primarily for their anticipated off-target effects because their efficacy is similar. Although copanlisib has demonstrated a higher response rate in a small number of patients, these drugs are generally comparable, said Dr. Smith. There are also no randomized data to guide clinical decision-making. "Selection of these agents is chosen based on the patient's likely tolerance and comorbidities," Dr. Smith explained. "Copanlisib can cause fatigue, hyperglycemia, hypertension, and diarrhea, which may be an issue for patients with certain preexisting conditions."

Importantly, no PI3K inhibitor is currently approved by the FDA for MZL, but there are recommendations in the NCCN Guidelines for B-Cell Lymphomas to consider use of these agents in appropriate patients. "It is my hope that with better understanding of the pathobiology of this disease, we'll have better treatment targets and emerging paradigms that allow patients a fixedduration therapy that is very effective administration," Dr. Smith concluded.

Disclosures: Dr. Smith has disclosed that he receives grant/research support from Acerta Pharma, AstraZeneca Pharmaceuticals LP, Bayer HealthCare, BeiGene, Denovo Biopharma, Genentech, Inc., Karyopharm Therapeutics, Merck \& Co., Inc., Pharmacyclics, and Portola Pharmaceuticals, Inc.; receives consulting fees from AstraZeneca Pharmaceuticals LP, Denovo Biopharma, Genentech, Inc., Kite Pharma, and Millennium Pharmaceuticals, Inc.; and is a scientific advisor for BeiGene. Dr. Smith also discloses that his spouse receives consulting fees from Ayala and Bristol-Myers Squibb, and grant/ research support from Ayala, Bristol-Myers Squibb Company, Ignyta, and Incyte Corporation.

Correspondence: Stephen D. Smith, MD, University of Washington/Fred Hutchinson Cancer Research Center, 617 Eastlake Avenue E, CE3-300, Seattle, WA 98109. Email: ssmith50@seattlecca.org

\section{References}

1. Rummel MJ, Niederle N, Maschmeyer G, et al. Bendamustine plus rituximab versus CHOP plus rituximab as first-line treatment for patients with indolent and mantle-cell lymphomas: an open-label, multicentre, randomised, phase 3 non-inferiority trial. Lancet 2013;381:1203-1210.

2. Baldini L, Brugiatelli M, Luminari $S$, et al. Treatment of indolent B-cell nonfollicular lymphomas: final results of the LL01 randomized trial of the Gruppo Italiano per lo Studio dei Linfomi. J Clin Oncol 2003;21:1459-1465.

3. Zucca E, Conconi A, Martinelli G, et al. Final results of the IELSG-19 randomized trial of mucosa-associated lymphoid tissue lymphoma: improved event-free and progression-free survival with rituximab plus chlorambucil versus either chlorambucil or rituximab monotherapy. J Clin Oncol 2017;35:1905-1912.

4. Salar A, Domingo-Domenech E, Panizo C, et al. First-line responseadapted treatment with the combination of bendamustine and rituximab in patients with mucosa-associated lymphoid tissue lymphoma (MALT2008-01): a multicentre, single-arm, phase 2 trial. Lancet Haematol 2014;1:e104-111.

5. Williams ME, Hong F, Gascoyne RD, et al. Rituximab extended schedule or retreatment trial for low tumour burden non-follicular indolent B-cell non-Hodgkin lymphomas: Eastern Cooperative Oncology Group Protocol E4402. Br J Haematol 2016;173:867-875.

6. Arcaini L, Rossi D, Paulli M. Splenic marginal zone lymphoma: from genetics to management. Blood 2016;127:2072-2081.
7. Kalpadakis C, Pangalis GA, Sachanas S, et al. Rituximab monotherapy in splenic marginal zone lymphoma: prolonged responses and potential benefit from maintenance. Blood 2018;132:666-670.

8. Noy A, de Vos $S$, Thieblemont $C$, et al. Targeting Bruton tyrosine kinase with ibrutinib in relapsed/refractory marginal zone lymphoma. Blood 2017;129:2224-2232.

9. Sacchi S, Marcheselli R, Bari A, et al. Safety and efficacy of lenalidomide in combination with rituximab in recurrent indolent non-follicular lymphoma: final results of a phase II study conducted by the Fondazione Italiana Linfomi. Haematologica 2016;101:e196-199.

10. Kiesewetter B, Willenbacher E, Willenbacher W, et al. A phase 2 study of rituximab plus lenalidomide for mucosa-associated lymphoid tissue lymphoma. Blood 2017;129:383-385.

11. Leonard JP, Trneny M, Izutsu K, et al. AUGMENT: a phase III study of lenalidomide plus rituximab versus placebo plus rituximab in relapsed or refractory indolent lymphoma. J Clin Oncol 2019;37: 1188-1199

12. Zelenetz AD, Gordon LI, Abramson JS, et al. NCCN Clinical Practice Guidelines in Oncology for B-Cell Lymphomas. Version 4.2020. Accessed October 19, 2020. Available at NCCN.org

13. Zucca E, Arcaini L, Buske C, et al. Marginal zone lymphomas: ESMO clinical practice guidelines for diagnosis, treatment and follow-up. Ann Oncol 2020;31:17-29. 